\title{
Spectrum Sensing Based On Deep Learning To Increase Spectrum Utilization
}

\section{Tae-Yun Jung and Eui-Rim Jeong*}

Graduate student, Department of Mobile Convergence and Engineering, Hanbat National University, Daejeon, 34158, Republic of Korea.

*Professor, Department of Information and Communication Engineering, Hanbat National University, Daejeon, 34158, Republic of Korea.

*Corresponding author.; Email address: erjeong@ hanbat.ac.kr

Article History:Received:11 november 2020; Accepted: 27 December 2020; Published online: 05 April 2021

\begin{abstract}
This paper proposes a new spectrum sensing technique for cognitive radio systems. To determine vacancy of the spectrum, the proposed method employs the recurrent neural network (RNN), one of the popular deep learning techniques. The proposed technique determines the spectrum occupancy of the primary user (PU) by observing the received signal's energy and any information on the PU signal characteristic is not used. To this end, the received signal's spectrum is obtained by fast Fourier transform (FFT). This process is performed on consecutive received signals and the resulting spectrums are stacked. Finally, a 2-dimensional spectrum (or spectrogram) is made. This 2-D spectrum is cut into sensing channel bandwidths and inputted to the deep learning model to decide the channel's occupancy. While the recently published spectrum sensing technique based on convolutional neural network $(\mathrm{CNN})$ relies on an empty channel, the proposed technique does not require any empty channel. Only the channel signal of interest to sense is needed. Since spectrum sensing results is two (busy or idle), binary classification deep learning model is developed. According to the computer simulation results, the proposed method has similar performance with the conventional CNN-based method while the spectral efficiency of the proposed method is much higher than that of the existing scheme. In addition, the overall learnable parameters of the proposed deep learning model is only $2 / 3$ of the existing method.
\end{abstract}

Keywords: Cognitive radio, Spectrum sensing, Energy detection, Deep learning, Binary classification.

\section{Introduction}

The cognitive radio (CR) is an effective mean to alleviate the problem of wireless frequency resource scarcity and underused licensed spectrum [1]. In CR systems, licensed users are called primary user (PU), and unlicensed users are called secondary user (SU). In the wireless communication systems, the spectrum allocated to the PU is not always used and the SU can share the idle spectrum. Many researches have been conducted to prevent the SU signal from interfering with the PU's signal. The objective of spectrum sensing is to determine spectrum occupancy by the PU accurately. This technique is grouped into three categories: match filter [2, 3], energy detection [4], and cooperative method [5]. For spectrum sensing, the most popular and simplest method is energy detection that determines PU existence based on the received energy. However, the energy detection requires the noise power and the sensing performance is highly sensitive to the accuracy of the estimated noise power. To overcome this sensitivity, various methods have been proposed in energy detection [6].

Recently, machine learning techniques are introduced for spectrum sensing in the industry [7]; artificial neural network (ANN) based spectrum sensing was proposed in [8], and deep learning techniques (e.g. convolutional neural network $(\mathrm{CNN})$ and recurrent neural network (RNN)) to accurately identify the spectrum occupancy were suggested in [9-11]. Especially, the technique in [11] is relatively simple to implement but shows good performance even at low signal to noise ratios (SNRs). However, this method requires one empty channel, and this requirement lowers the spectral efficiency of both PU and SU.

This paper considers a new deep learning based spectrum sensing technique in the category of energy detection without empty channel assumption. The proposed one is a simple deep learning method that detects the presence / absence of PU signal just by observing sensing channel's energy. Specifically, the received signal is converted into a frequency spectrum vector through fast Fourier transform (FFT). Performing this process on consecutive received signals and stacking the resulting spectrums, 2-dimensional spectrum is made. Then, this 2-D spectrum is cut by a sensing channel bandwidth and inputted to the CNN classifier to decide the channel's occupancy. The performance of the proposed spectrum sensor is verified through computer simulation using Matlab. According to the results, the spectrum sensing accuracies of the proposed technique and the existing method in [11] are similar. However, since the proposed method does not need to empty one PU channel, the

*Corresponding author: Eui-Rim Jeong

538

Professor, Department of Information and Communication Engineering, Hanbat National University, Daejeon,

34158, Republic of Korea.

Email address: erjeong@ hanbat.ac.kr 
spectral efficiency is higher than that of the existing scheme. In addition, the overall learnable parameters of the proposed deep learning model is only $2 / 3$ of the existing model.

\section{System model}

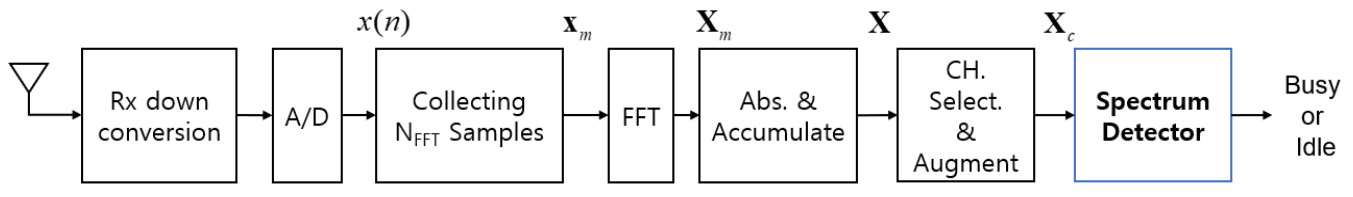

Figure 1 System model

The system model considered in this paper is in Figure 1. The received signal is converted into a baseband digital signal by radio frequency (RF) down converter and analog to digital converter (ADC). This signal is denoted by $x(n)$. A total $N_{F F T}$, the size of the FFT, and collects samples by overlapping as $N_{0}$. This process can reduce the time to collect the entire received signal. $\mathrm{B}$ is the total number of signal blocks observed. The collected signal blocks are as follows.

$$
\boldsymbol{x}_{m}=\left[r\left(m N_{0}\right), r\left(m N_{0}+1\right), \cdots, r\left(m N_{0}+N_{F F T}-1\right)\right] \text { for } m=0, \cdots, B-1
$$

$X_{m}$ conducts an FFT to convert to a frequency domain. The size of $\widehat{\boldsymbol{X}}_{m}$ is $N_{F F T} \times 1$.

$$
\widehat{\boldsymbol{X}}_{m}=F F T\left\{\boldsymbol{x}_{m}\right\}=\left[X_{m}(0), X_{m}(1), \cdots, X_{m}\left(N_{F F T}-1\right)\right]^{T}
$$

For spectrum sensing, absolute values are taken to yield.

$$
\left|\widehat{\boldsymbol{X}}_{m}\right| \triangleq\left[\left|\widehat{\boldsymbol{X}}_{m}(0)\right|,\left|\widehat{\boldsymbol{X}}_{m}(1)\right|, \cdots,\left|\widehat{\boldsymbol{X}}_{m}\left(N_{F F T}-1\right)\right|\right]^{T}
$$

For consecutive received signals, the process (1) - (3) are performed the resulting B vectors of $\left|\widehat{\boldsymbol{X}}_{m}\right|$ for $0 \leq$ $m<B$ are stacked. The stacked signal becomes a 2-D matrix denoted by $\boldsymbol{X} \in R^{N_{F F T} \times B}$ :

$$
\boldsymbol{X}=\left\lfloor\left|\hat{X}_{0}\right|,\left|\hat{X}_{1}\right|, \cdots,\left|\hat{X}_{B-1}\right|\right]
$$

The 2-D signal $\boldsymbol{X}$ is made by FFT of broadband signal and can include multiple PU channels. To sense by PU channel interval, it must be separated into sensing bandwidth intervals. Suppose the whole bandwidth of the receiving signal corresponds to $N_{F F T}$ and the bandwidth of sensing channel is $K_{C}$. The total number of sensing channels is $N_{F F T} / K_{C}$. If the sampling clock of the ADC is $F_{S}(\mathrm{~Hz})$, the bandwidth of each channel is $K_{C} F_{S} / N_{F F T}(\mathrm{~Hz})$. The existing research in [11] assumes that the last channel is always empty and is not allocated by the PU. [11] insists that the empty channel (or noise-only channel) is very advantageous for spectrum sensing.

$$
\boldsymbol{X}_{c}=\left[\begin{array}{c}
\widetilde{\boldsymbol{X}}_{c} \\
\widetilde{\boldsymbol{X}}_{N_{F F T} / K_{C^{-1}}}
\end{array}\right] \text { for } c=0, \cdots, N_{F F T} / K_{C}-2
$$

$\widetilde{\boldsymbol{X}}_{c}$ is of size $K_{C} \times B$ and is a submatrix of $\boldsymbol{X}$ and is a matrix made by selecting rows from $c K_{C}+1$ to $(c+1) K_{C}$. In (5), $\widehat{\boldsymbol{X}}_{N_{F F T} / K_{C}-1}$ is the last sensing channel and can be regarded as a noise vector because the last channel is always unassigned. As a result, the conventional work uses a matrix $\left(\boldsymbol{X}_{c}\right)$ of size $2 K_{C} \times B$ to determine the presence of PU signals.

\section{Conventional spectrum sensing}

This section introduces the conventional spectrum sensing technique using a 2-dimensional matrix $\boldsymbol{X}_{c}$. First, the threshold-based spectrum detector in introduced, and then a deep learning-based spectrum detector to be compared is explained.

\subsection{Threshold-based spectrum detector}

The conventional energy detection method estimates the power of noise and obtains threshold value based on this. After that, when the signal if PU signal exceeds the threshold value, it decides that it exists.

$$
\left\{\begin{array}{l}
\text { Busy, } \hat{P}_{c}>\eta \\
\text { Idle, otherwise }
\end{array}\right.
$$

$\eta$ is the threshold value as follows:

$$
\eta=\alpha \times \hat{P}_{n}
$$


$\alpha$ is a positive real number value. $\hat{P}_{n}$ is the estimated value of noise power and is obtained by the last channel $\widetilde{\boldsymbol{X}}_{N_{F F T} / K_{C}-1}$.

$$
\widehat{\mathrm{P}}_{\mathrm{n}}=\frac{1}{\mathrm{~K}_{\mathrm{C}} \mathrm{B}} \sum_{\mathrm{n}=0}^{\mathrm{B}-1} \sum_{\mathrm{m}=0}^{\mathrm{K}_{\mathrm{C}}-1}\left|\widetilde{\mathbf{X}}_{\mathrm{N}_{\mathrm{FFT}} / \mathrm{K}_{\mathrm{C}}-1}(\mathrm{~m}, \mathrm{n})\right|^{2}
$$

In Eq. (8), $\hat{P}_{c}$ means the estimated power value of the PU channel and is obtained as follows:

$$
\hat{P}_{c}=\frac{1}{K_{C} B} \sum_{n=0}^{B-1} \sum_{m=0}^{K_{C}-1}\left|\tilde{X}_{c}(m, n)\right|^{2}
$$

The threshold-based detector performs well when the power of the noise signal is accurately estimated. However, in the opposite case, performance deviations are severe. In addition, there is a problem in that the SNR value must be known in advance for spectrum sensing.

\subsection{CNN-based spectrum detector [10]}

The existing CNN-based spectrum detector is briefly explained. On the left side of Figure $2, \boldsymbol{X}$ is shown as a black-and-white image when $N_{F F T}=512, B=64$. White is a large signal, black is a small signal, and the middle value is gray. This figure is obtained when $S N R=20[\mathrm{~dB}]$, so the presence of the PU signal is clearly distinguishable. The CNN based spectrum detector in [10] has $\boldsymbol{X}_{c}$ as the input and consists of three convolutional layers. Each convolution layer includes a batch normalization layer, a pooling layer. The last convolution layer excludes the pooling layer. The channel size (or number of filters) in each convolution layer is 8,16 , and 32 , the filter size is $3 \times 3$, and the stride is 1 . The pooling layer is max pooling with stride 2 . The activation function is ReLU (Rectified Linear Unit). The output of the last convolution layer goes through the fully connected layer and finally determines the presence of the PU signal at the classification layer.

\subsection{RNN-based spectrum detector [11]}

The existing RNN structure in [11-13] is shown on the right side of Figure 2. For spectrum sensing, the RNN model also uses $\boldsymbol{X}_{c}$ as the input, but at each time, the column vectors of $\boldsymbol{X}_{c}$ are inputted one by one. Therefore, input to the RNN model is a total B column vectors with size $2 K_{C} \times 1$. The first input to the LSTM (Long short-term memory) cell is $\boldsymbol{x}_{c, 0}=\left[\boldsymbol{X}_{c}(0,0), \cdots, \boldsymbol{X}_{c}\left(2 K_{C}-1,0\right)\right]^{T}$; the next input is $\boldsymbol{x}_{c, 1}=$ $\left[\boldsymbol{X}_{c}(0,1), \cdots, \boldsymbol{X}_{c}\left(2 K_{C}-1,1\right)\right]^{T}$ and so on. The structure of the RNN model consists of LSTM cells, the fully connected layer, and the binary classification layer. The number of LSTM cells is 64 . The final output value after the last vector $\left(\boldsymbol{x}_{C, B-1}\right)$ is Busy or Idle.

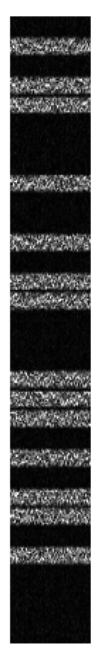

$X$
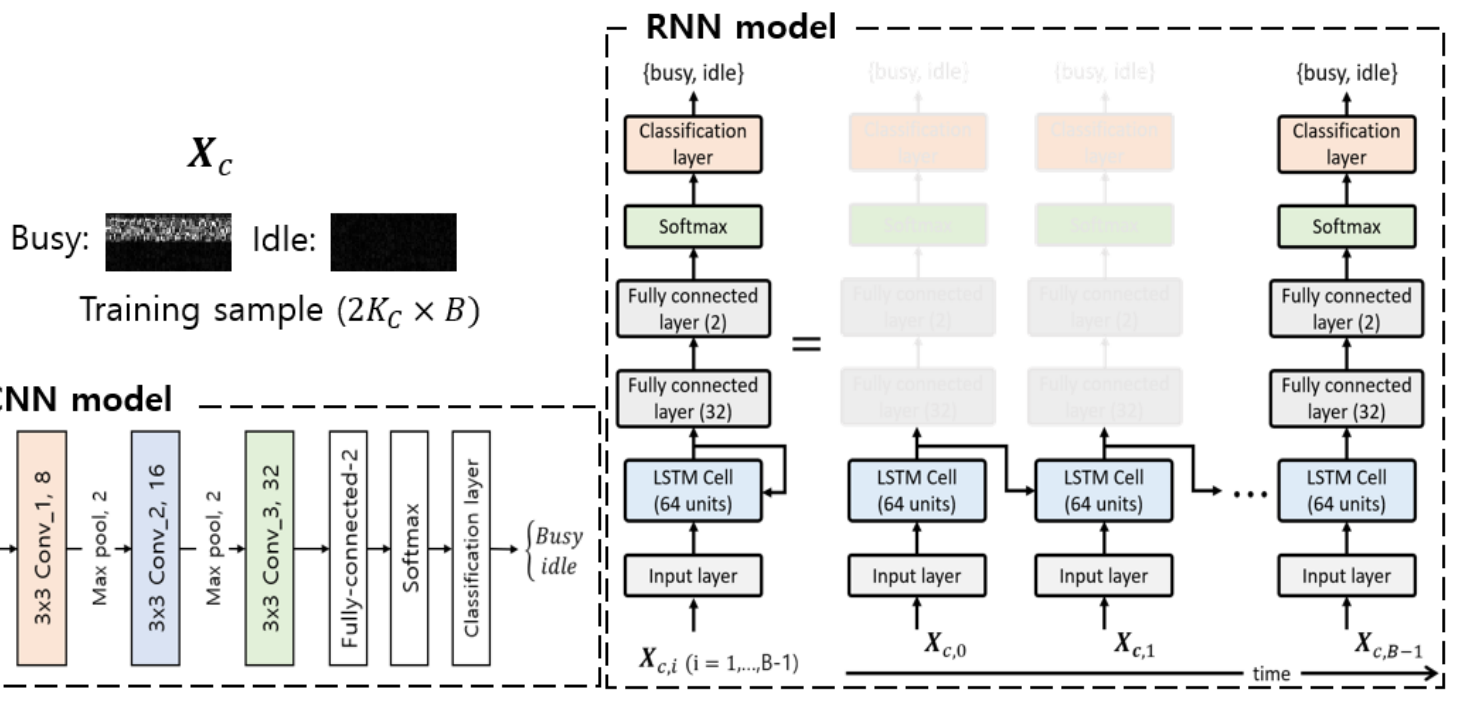

Figure 2 Block diagrams of conventional techniques in [10] and [11] when B = 64

\section{Proposed spectrum sensing}

This section introduces the proposed CNN-based spectrum sensing technique. The proposed method is the same as the conventional spectrum detector structure. However, the input signal of the deep learning model is 
different from the conventional techniques in [10] and [11] to improve spectral efficiency. Figure 3 (a) shows the typical PU signal's spectrum. As can be seen, PU signal has a guard band to prevent interference between adjacent channels. It can be seen that there is only noise in the guard band. Therefore, instead of using additional intentional noise channel, the PU's single channel of interest may be sufficient to determine spectrum usage. This is the motivation of the proposed technique. Figure 3 (b) shows $\widetilde{\boldsymbol{X}}_{c}$ without noise channel in (5). When the channel is busy, the far upper and lower region look black because of the guard band. The input of the proposed detector is a 2-dimensional matrix $\widetilde{\boldsymbol{X}}_{c}$ in size $K_{C} \times B$. Existing researches must assume an empty channel to ensure the performance of spectrum sensing. However, this is a major disadvantage due to spectral efficiency loss. In contrast, the proposed technique can relieve from this problem.

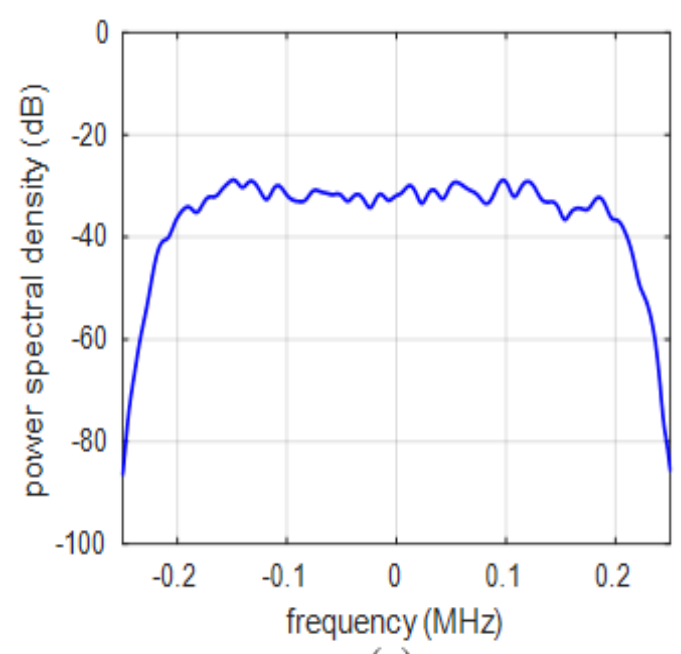

(a)

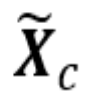

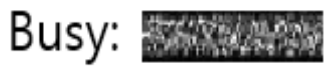

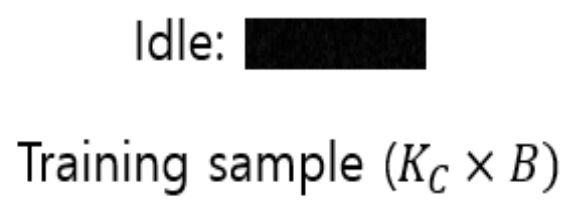

(b)

Figure 3 (a) PU signal's spectrum (b) Input signal for the proposed spectrum detector

\section{Simulation results}

\subsection{Environment and signal preparation}

To access the performance of the proposed technique, computer simulation is conducted using MATLAB R2020a. The sampling frequency of the received signal is $F_{S}=16 \mathrm{MHz}$ and the bandwidth of a sensing channel is $0.5 \mathrm{MHz}$. The number of channels that can be observed at a time through broadband sampling is 32 $(16 M / 0.5 M)$. The parameters used in the proposed method are shown in Table 1. The length of the observation $\operatorname{signal} \operatorname{block}(B)$ is fixed at 64 .

Table1 Parameters for spectrum sensing

\begin{tabular}{cc}
\hline Parameter & Value \\
\hline Sampling clock & $F_{S}=16 \mathrm{MHz}$ \\
\hline Channel bandwidth & $0.5 \mathrm{MHz}$ \\
\hline FFT size & $N_{F F T}=512$ \\
\hline Overlap length & $N_{0}=N_{F F T} / 2$ \\
\hline Sensing channel bandwidth in FFT points & $K_{C}=16$ \\
\hline Number of observation signal blocks & $B=64$ \\
\hline
\end{tabular}

The data sets for training the proposed deep learning model are generated with the same condition in [10] and [11]. The SNR of the training data is selected rendomly from $-20 \mathrm{~dB}$ to $50 \mathrm{~dB}$ and we produces a total of 620,000 data sets. The test data is generated with a SNR interval of 2 [dB] and 124,000 data sets are generated at each SNR. Training is performed to minimize cross-entropy and the optimization technique is SGDM (Stochastic Gradient Descent with Momentum). The learning rate is 0.001 and the batch size is 620. Figure 4 
shows the learning curve, and (a) is the proposed CNN model (b) is the proposed RNN model. In Figure 4. The learning curve is converged sufficiently without the need for a noise channel. In order to examine the performance of the proposed spectrum sensing technique, two indicators are measured. First is the MDR (miss detection ratio). Miss detection means the PU channel is empty but detected to be in use. Second is FDR (false detection ratio). False detection means the opposite of MDR. In spectrum sensing, it is important to minimize FDR to reduce interference from the SU to the PUs.

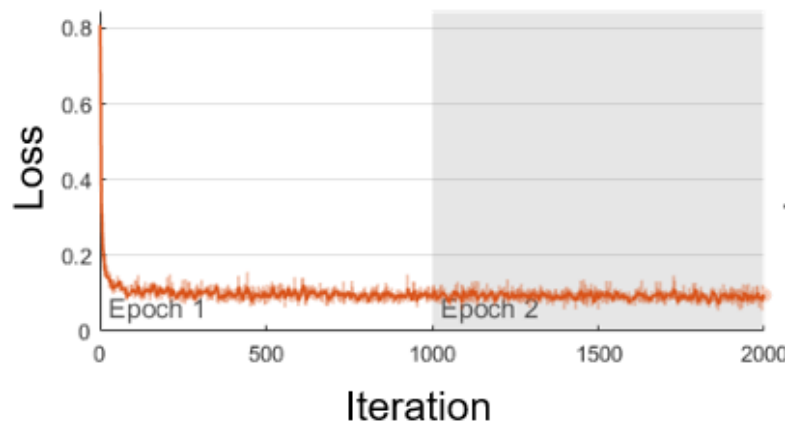

(a)

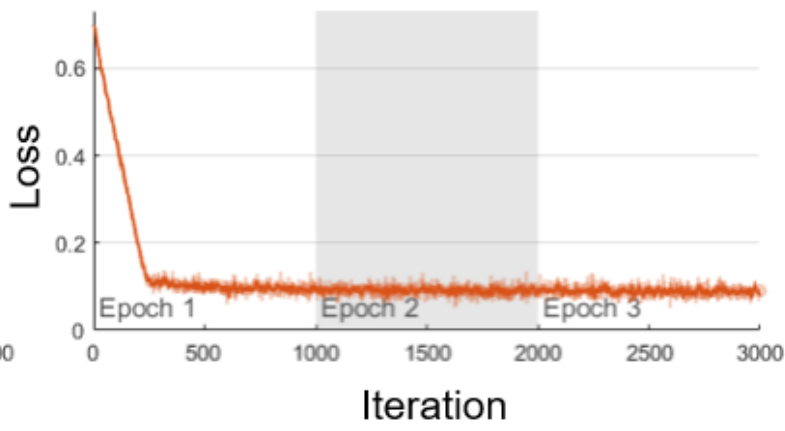

(b)

Figure 4 Learning curves for (a) proposed CNN model (b) proposed RNN model

\subsection{Results}

Figure 5 compares the performance of the CNN model. The test data to compare performance is all the same. $\eta$ of the threshold-based detector is determined by the formula (7), and $\alpha$ is selected optimally at each SNR. The results of the simulation show that the performance of the CNN model is almost identical regardless of the use of extra noise only channel. However, threshold-based method have a performance degradation about 2 [dB]. Figure 6 shows the performance of the RNN model and is also similar to the previous results (CNN model). Those results indicate that by using the proposed CNN or RNN spectrum detector, good spectrum sensing performance can be achieved without any spectrum resource waste due to the intentional empty channel.

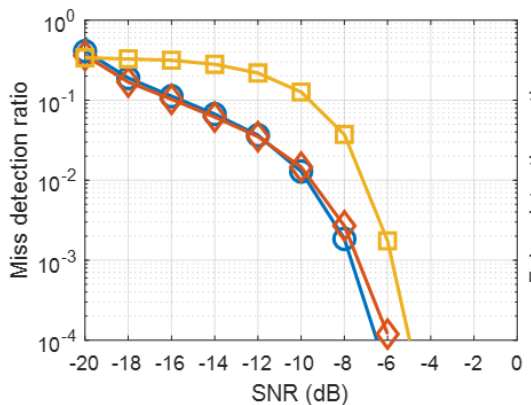

(a)

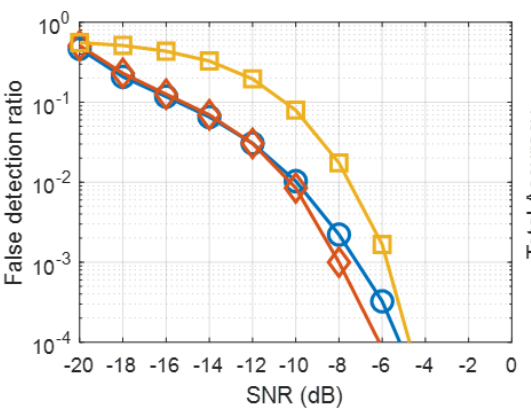

(b)

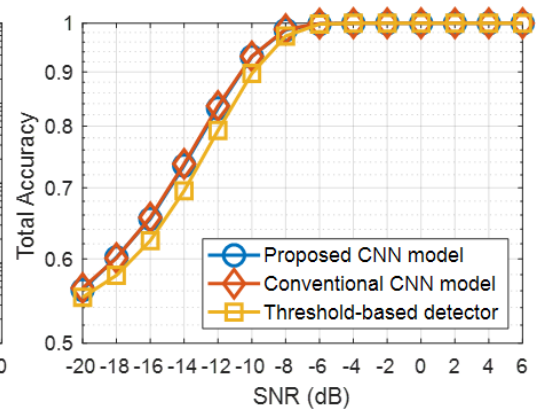

(c)

Figure 5 Simulation results with CNN model (a) MDR (b) FDR (c) ACC

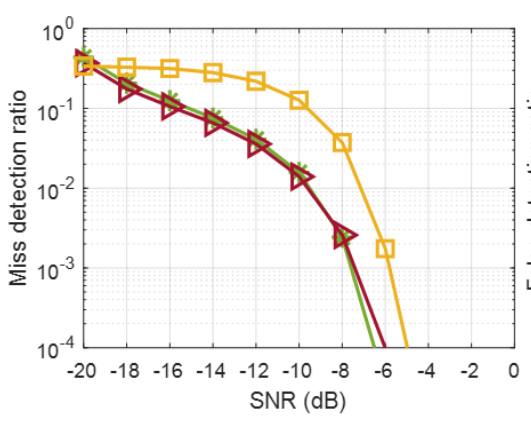

(a)

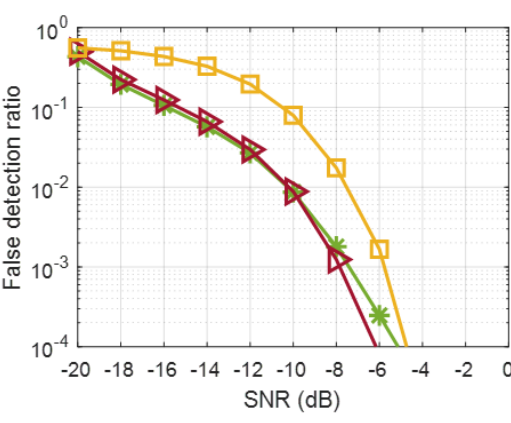

(b)

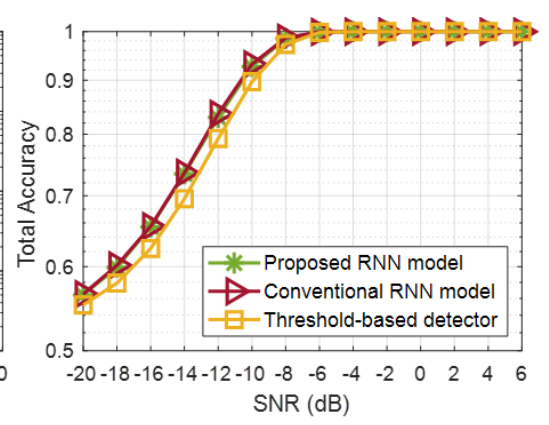

(c)

Figure 6 Simulation results with RNN model (a) MDR (b) FDR (c) ACC

\section{Conclusions}

This paper proposed a CNN-based spectrum sensing technique for sharing the PU's licensed spectrum. The 
proposed method was designed based on existing research and the use of the empty channel is removed. The simulation results show that the conventional and the proposed techniques have almost the same FDR and MDR performances. Those results indicate that by using the proposed technique, there is no spectrum resource waste to achieve reliable spectrum sensing performance.

\section{References}

1. Mansi S, Gajanan B. Spectrum sensing techniques in cognitive radio networks: A survey. International Journal of Next-Generation Networks. 2011;3(2):37-51.

2. Shobana S, Saravanan R, Muthaiah R. Matched filter based spectrum sensing on cognitive radio for OFDM WLANs. International Journal of Engineering and Technology. 2013;5(1):142-6.

3. Vadivelu R, Sankaranarayanan K, Vijayakumari V. Matched filter based spectrum sensing for cognitive radio at low signal to noise ratio. Journal of Theoretical and Applied Information Technique. 2015;62(1):107-113.

4. Ye Y, Li Y, Lu G, Zhou F. Improved energy detection with Laplacian noise in cognitive radio. IEEE Systems Journal. 2019;13(1):18-29.

5. Lee W, Kim M, Cho D-H. Deep cooperative sensing: cooperative spectrum sensing based on convolutional neural networks. IEEE Transactions on Vehicular Technology. 2019;68(3):3005-9.

6. Zeng Y, Liang Y-C, Zhang Rui. Blindly combined energy detection for spectrum sensing in Cognitive radio. IEEE Signal Processing Letters. 2008;15:649-652.

7. Zhang L, Xiao M, Wu G, Alam M, Liang Y-C, Li S. A survey of advanced techniques for spectrum sharing in 5G networks. IEEE Wireless Communications. 2017;24(5):44-51.

8. Saber M, Rharras AE, Saadane R, Aroussi HK, Wahbi M. Artificial neural networks, support vector machine and energy detection for spectrum sensing based on real signals. International Journal of Communication Networks and Information Security. 2019;11(1):52-60.

9. Zheng S, Chen S, Qi P, Zhou H, Yang X. Spectrum sensing based on deep learning classification for cognitive radios. China Communications. 2020;17(2):138-148.

10. Jung T-Y, Lee E-S, Kim D-K, Oh J-M, Noh W-Y, Jeong E-R. CNN based spectrum sensing technique for cognitive radio communications. Journal of the Korea Institute of Information and Communication Engineering. 2020;24(2):276-284. Available from: https://www.kci.go.kr/kciportal/ci/sereArticleSearch/ciSereArtiView.kci?sereArticleSearchBean.artiId= ART002560693

11. Jung T-Y, Jeong E-R. Recurrent Neural Network Based Spectrum Sensing Technique for Cognitive Radio Communications. Journal of the Korea Institute of Information and Communication Engineering. 2020;24(6):759-767. Available from: https://www.kci.go.kr/kciportal/ci/sereArticleSearch/ciSereArtiView.kci?sereArticleSearchBean.artiId= ART002598091

12. Mallick, P. K., Mishra, D., PATANAIK, S., \& Shaw, K. (2016). A novel supervised gene clustering approachby mining interdependent gene patterns. International Journal of Pharma and Bio Sciences, 7(4).

13. Samal, A. K., Mallick, P. K., Pramanik, J., Pani, S. K., \& Jelli, R. (2019). Functional link artificial neural network (FLANN) based design of a conditional branch predictor. In Cognitive Informatics and Soft Computing (pp. 131-152). Springer, Singapore. 